\title{
Cardiovascular disease risk in Irish adults
}

\author{
M. Buffini ${ }^{1}$, C.B. O’Donovan ${ }^{1}$, A.P. Nugent ${ }^{1}$, J. Walton ${ }^{2}$, A. Flynn ${ }^{2}$ and B.A. McNulty ${ }^{1}$ \\ ${ }^{1}$ Institute of Food and Health, University College Dublin, Ireland and ${ }^{2}$ School of Food and Nutritional Sciences, \\ University College Cork, Ireland
}

Cardiovascular disease (CVD) accounts for $36 \%$ of all deaths per year in Ireland, making it the most common cause of death ${ }^{(1)}$. The association between certain nutrients and CVD is well established, however recent research suggests that an overall dietary pattern may be a better predictor of CVD risk $^{(2)}$. The aim of the current study was to identify CVD risk within a sub cohort $(\mathrm{n}=754)$ of the National Adult Nutrition Survey (NANS) (www.iuna.net). The NANS was a cross-sectional food consumption survey carried out between 2008 and 2010 in an Irish adult population $(n=1500)$, a four day semi-weighed food diary was used to collect habitual food and beverage intakes, and $79 \%$ of participants provided blood samples. Stepwise regression analysis was used to identify certain markers of metabolic health as having a significant effect on the SCORE risk factors, total cholesterol, HDL cholesterol and systemic blood pressure. SCORE is a commonly used tool for identifying those at risk of CVD ${ }^{(3)}$. These markers were used to perform cluster analysis of NANS participants split by gender.

\begin{tabular}{|c|c|c|c|c|c|c|c|c|c|c|}
\hline \multirow[b]{3}{*}{ Clustering Variable } & \multicolumn{4}{|c|}{ Males } & \multicolumn{6}{|c|}{ Females } \\
\hline & \multicolumn{2}{|c|}{ Cluster $1(n=172)$} & \multicolumn{2}{|c|}{ Cluster $2(n=204)$} & \multicolumn{2}{|c|}{ Cluster $1(n=101)$} & \multicolumn{2}{|c|}{ Cluster $2(n=90)$} & \multicolumn{2}{|c|}{ Cluster $3(n=187)$} \\
\hline & Mean & $\mathrm{SD}$ & Mean & SD & Mean & SD & Mean & SD & Mean & SD \\
\hline Age (yrs) & 48.47 & $15 \cdot 25$ & $34.58 *$ & $16 \cdot 00$ & $52 \cdot 08^{\mathrm{a}}$ & $14 \cdot 00$ & $54 \cdot 77^{\mathrm{a}}$ & $14 \cdot 54$ & $33 \cdot 39^{\mathrm{b}}$ & $12 \cdot 08$ \\
\hline BM I $\left(\mathrm{kg} / \mathrm{m}^{2}\right)$ & 29.56 & $4 \cdot 31$ & $25 \cdot 54^{*}$ & 3.58 & $31.60^{\mathrm{a}}$ & $4 \cdot 55$ & $24 \cdot 78^{\mathrm{b}}$ & $3 \cdot 19$ & $24 \cdot 35^{\mathrm{b}}$ & $3 \cdot 31$ \\
\hline Weight $(\mathrm{kg})$ & $91 \cdot 24$ & $15 \cdot 36$ & $80 \cdot 68^{*}$ & $12 \cdot 10$ & $81.73^{a}$ & $12 \cdot 10$ & $64 \cdot 70^{\mathrm{b}}$ & 9.08 & $65.48^{b}$ & 9.04 \\
\hline Total Cholesterol $(\mathrm{mmol} / \mathrm{L})$ & $\mathbf{5 \cdot 5 0}$ & 0.88 & $4 \cdot 28 *$ & 0.74 & $5 \cdot 31^{\mathrm{a}}$ & 0.91 & $\mathbf{5 . 7 4}^{\mathrm{b}}$ & 0.97 & $4 \cdot 58^{\mathrm{c}}$ & 0.75 \\
\hline HDL Cholesterol (mmol/L) & $1 \cdot 34$ & $0 \cdot 31$ & $1.45 *$ & 0.33 & $1.47^{\mathrm{a}}$ & $0 \cdot 30$ & $2 \cdot 19^{\mathrm{b}}$ & $0 \cdot 42$ & $\overline{1.64^{\mathrm{c}}}$ & $0 \cdot 35$ \\
\hline ApoAl $(\mathrm{mg} / \mathrm{dl})$ & 147.46 & $29 \cdot 30$ & $148 \cdot 50$ & 31.86 & $15 \overline{7 \cdot 28^{a}}$ & $31 \cdot 30$ & $199.73^{\mathrm{b}}$ & $30 \cdot 68$ & $154 \cdot 43^{\mathrm{a}}$ & 41.72 \\
\hline ApoB (mg/dl) & $127 \cdot 17$ & $23 \cdot 86$ & $85 \cdot 67^{*}$ & $22 \cdot 30$ & $123 \cdot 27^{\mathrm{a}}$ & $26 \cdot 76$ & $113 \cdot 71^{\mathrm{b}}$ & $25 \cdot 82$ & $89.51^{\mathrm{c}}$ & 21.81 \\
\hline ApoC2 (mg/dl) & $6 \cdot 60$ & 2.78 & $3 \cdot 55^{*}$ & 1.63 & $5 \cdot 37^{\mathrm{a}}$ & $2 \cdot 27$ & $5 \cdot 35^{\mathrm{a}}$ & $2 \cdot 10$ & $3 \cdot 11^{\mathrm{b}}$ & 1.54 \\
\hline ApoC3 (mg/dl) & $11 \cdot 71$ & 3.77 & $7 \cdot 44^{*}$ & $2 \cdot 56$ & $10 \cdot 52^{\mathrm{a}}$ & 2.98 & $11.29^{\mathrm{a}}$ & 4.03 & $7 \cdot 19^{b}$ & 2.59 \\
\hline ApoE (mg/dl) & $\mathbf{3 \cdot 2 7}$ & $1 \cdot 13$ & $2.46^{*}$ & 0.94 & $3 \cdot 18^{\mathrm{a}}$ & $1 \cdot 17$ & $3.96^{\mathrm{b}}$ & 1.44 & $2 \cdot 50^{\mathrm{c}}$ & 0.94 \\
\hline Adiponectin $(\mu \mathrm{g} / \mathrm{ml})$ & $4 \cdot 38$ & $2 \cdot 10$ & $5 \cdot 06^{*}$ & 1.91 & $5.09^{\mathrm{a}}$ & 1.89 & $10 \cdot 97^{\mathrm{b}}$ & 4.68 & $6 \cdot 89^{c}$ & $2 \cdot 38$ \\
\hline Glucose $(\mathrm{mmol} / \mathrm{L})$ & 5.55 & 0.85 & $5 \cdot 07 *$ & 0.56 & $5.62^{a}$ & 0.93 & $5 \cdot 01^{\mathrm{b}}$ & $0 \cdot 56$ & $4 \cdot 86^{\mathrm{b}}$ & $0 \cdot 51$ \\
\hline $\mathrm{TNF}-\alpha(\mathrm{pg} / \mathrm{ml})$ & $7 \cdot 17$ & $2 \cdot 11$ & $6 \cdot 83$ & 1.78 & $7.08^{\mathrm{a}}$ & 1.79 & $5.99^{\mathrm{b}}$ & 1.71 & $\overline{6.19}^{\mathrm{b}}$ & $2 \cdot 00$ \\
\hline Systolic BP (mmHg) & 135.44 & $16 \cdot 51$ & $125 \cdot 17^{*}$ & 13.47 & $127 \cdot 24^{\mathrm{a}}$ & $15 \cdot 80$ & $123 \cdot 47^{\mathrm{a}}$ & $20 \cdot 06$ & $110.65^{\mathrm{b}}$ & 12.44 \\
\hline
\end{tabular}

Significant differences between male clusters were analysed with an unpaired t-test $(\mathrm{p}<0 \cdot 05) .{ }^{*}$ Denotes statistical significance be-

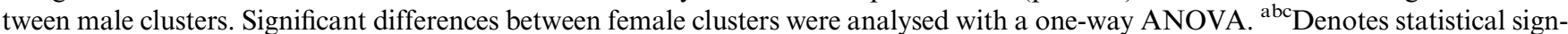
ificance between female clusters $(\mathrm{p}<0.05)$. Bolded text represents highest values. Underlined text represents lowest values. BMI body mass index, Apo apolipoprotein, HDL high density lipoprotein, BP blood pressure, TNF- $\alpha$ tumour necrosis factor alpha.

Two clusters were identified in the male cohort. Cluster 1 were older and had significantly higher BMIs, weight, systolic blood pressure, and blood lipids $(\mathrm{p} \leqslant 0.001)$. Cluster 2 members had higher levels of adiponectin and HDL cholesterol. In the female cohort three clusters were identified. Cluster 3 were the youngest $(\mathrm{p}<0.001)$, however they did not differ from cluster 2 in terms of BMI, weight, blood glucose or TNF- $\alpha$. Females in clusters $1 \& 2$ were similar in age, however those in cluster one had significantly higher BMIs, weight, blood glucose, systolic BP and TNF- $\alpha(p<0 \cdot 001)$. Cluster 2 members had the overall highest total cholesterol $(p<0 \cdot 001)$ which corresponded with the overall highest HDL cholesterol levels $(p<0.001)$. There was no difference in smoking status between cluster for males $(p=0 \cdot 36)$ or females $(p=0 \cdot 10)$. Further analysis will determine differences in the dietary patterns of cluster members by examining food group and nutrient intakes and to establish if the dietary patterns of those at greater risk can explain greater susceptibility to CVD.

This project was funded by Department of Agriculture, Food and the Marine under the Nutridata project (2013-2016).

1. HSE Report (2006) Available at: https://www.irishheart.ie/media/pub/heartwise/2006/changing_pattern_of_cvd_in_ireland.pdf

2. Kerver JM, Yang EJ, Bianchi L et al. (2003) Am J Clin Nutr 78 (6) 1103-1110

3. Conroy RM, Pyorala K, Fitzgerald AP et al. (2003) Eur Heart J 24 (11) 987-1003 